\title{
The role of complex IV activity in the axonal response of mitochondria to demyelination in experimental disease models of multiple sclerosis
}

Simon Licht-Mayer ${ }^{1}$, Graham R. Campbell ${ }^{1}$, Arpan R. Mehta ${ }^{1,2}$, Katie McGill ${ }^{1}$, Alex Symonds $^{1}$, Sarah Al-Azki ${ }^{3}$, Gareth Pryce ${ }^{3}$, Stephanie Zandee ${ }^{4}$, Chao Zhao ${ }^{5}$, Markus Kipp ${ }^{6}$, Kenneth J Smith ${ }^{7}$, David Baker ${ }^{3}$, Daniel Altmann ${ }^{8}$, Stephen M. Anderton ${ }^{4}$, Yolanda S. Kap ${ }^{9}$, Jon D. Laman',10, Bert A. 't Hart' ${ }^{9-11}$, Moses Rodriguez'12, Robin J.M. Franklin' ${ }^{5}$, Siddharthan Chandran $^{1,2}$, Hans Lassmann ${ }^{13}$, Bruce D. Trapp ${ }^{14}$ and Don J. Mahad ${ }^{* 1}$

${ }^{1}$ Centre for Clinical Brain Sciences, University of Edinburgh, Chancellor's Building, 49 Little France Crescent, Edinburgh, EH16 4SB, UK

${ }^{2}$ UK Dementia Research Institute, University of Edinburgh, Edinburgh, UK

${ }^{3}$ Blizard Institute, Barts and The London School of Medicine and Dentistry, Queen Mary University of London, 4 Newark Street, London E1 2AT, UK

${ }^{4}$ Centre for Inflammation Research, University of Edinburgh, 47 Little France Crescent, Edinburgh, EH16 4SB, UK

${ }^{5}$ Wellcome Trust-MRC Cambridge Stem Cell Institute, Jeffrey Cheah Biomedical Centre, University of Cambridge, Cambridge Biomedical Campus, Cambridge CB2 OAW, UK

${ }^{6}$ Institute of Anatomy, Rostock University Medical Center, Gertrudenstrasse 9, 18057 Rostock, Germany

${ }^{7}$ Department of Neuroinflammation, The UCL Queen Square Institute of Neurology, University College London, 1 Wakefield Street, London, WC1N 1PJ, UK

${ }^{8}$ Faculty of Medicine, Department of Medicine, Hammersmith Campus, London, UK

${ }^{9}$ Department of Immunobiology, Biomedical Primate Research Centre, Rijswijk, The Netherlands

${ }^{10}$ Dept. Pathology and Medical Biology and MS Center Noord Nederland (MSCNN), University Groningen, University Medical Center Groningen, The Netherlands

${ }^{11}$ Dept. Anatomy and Neuroscience, Amsterdam University Medical Center (V|UMC|), Amsterdam, Netherlands

${ }^{12}$ Department of Neurology and Immunology, Mayo College of Medicine and Science, Rochester, Minnesota MN55905, USA 
${ }^{13}$ Department of Neuroimmunology, Center for Brain Research, Medical University Vienna, Spitalgasse 4, 1090, Vienna, Austria

${ }^{14}$ Department of Neuroscience, Lerner Research Institute, Cleveland Clinic, Cleveland Ohio OH44195, USA

Key words: mitochondria, experimental demyelination, axon injury, multiple sclerosis and neuroprotection

*Correspondence to: $\operatorname{Dr}$ D J Mahad, ${ }^{1}$ Centre for Clinical Brain Sciences, University of Edinburgh, Chancellor's Building, 49 Little France Crescent, Edinburgh, EH16 4SB, UK

Email: don.mahad@ed.ac.uk Tel +44 131 2426164. Fax +44 1912228553

$\dagger$ †oint first authors. 


\begin{abstract}
Axon loss is a cardinal neuropathological feature of multiple sclerosis (MS). Axonal injury in MS and experimental disease models is most frequently detected in acutely demyelinating regions. Furthermore, acute axonal injury consistently correlates with the extent of inflammatory demyelination. Following lysolecithin-induced demyelination, we recently reported a compensatory response in neurons, where mitochondria move from the cell body to the acutely demyelinated axon and increase the mitochondrial content. We termed this energetics phenomenon, that is also evident in MS, the axonal response of mitochondria to demyelination (ARMD). In the present study, we assessed axonal mitochondrial content as well as axonal mitochondrial respiratory chain complex IV activity (COX) of axons and related these to axonal injury in nine different experimental disease models. We consistently found ARMD in all experimental disease models. However, the increase in mitochondrial content within demyelinated axons was not always accompanied by a proportionate increase in COX activity, particularly in experimental autoimmune encephalomyelitis (EAE). Axonal COX activity inversely correlated with the extent of axonal injury in experimental disease models. Our findings indicate that ARMD is a consistent and prominent finding and emphasises the need to preserve axonal mitochondrial COX activity in inflammatory demyelination, paving the way for the development of novel neuroprotective therapies.
\end{abstract}




\section{Introduction}

Axonal loss is a cardinal neuropathological feature of multiple sclerosis (MS) $[9,27]$. Axonal injury is most prominently observed in actively demyelinating regions of MS and a gathering body of evidence implicates a state of energy failure in the degeneration of demyelinated axons [14]. The bioenergetic need (in terms of ATP) of demyelinated axons is said to be increased owing to the redistribution of ion channels [28]. In keeping with this increase in energy demand by demyelinated axons, we recently identified a neuronal compensatory mechanism where mitochondria move from the cell body to the acutely demyelinated axon, increasing the axonal mitochondrial content and energy producing capacity [13]. We termed this homeostatic mechanism the axonal response of mitochondria to demyelination (ARMD) [13].

Increased axonal mitochondrial content, reflecting ARMD, has been reported in MS and in a limited number of experimental disease models [17, 19, 24]. In MS, the increase in mitochondrial content of non-degenerated demyelinated axons was accompanied by increased axonal mitochondrial respiratory chain complex IV activity [15, 29, 32]. At the edge of chronic active MS lesions, where axon transection is most prominent, axonal complex IV activity inversely correlated with the extent of inflammation [15]. In inflammatory demyelinating environments, axonal mitochondrial function is compromised, perhaps by nitric oxide, an inhibitor of complex IV, and mitochondria may be damaged by post translational modification of respiratory chain complex subunits due to nitration [21, 25]. Furthermore, axonal mitochondrial transport may be perturbed by inflammation, as evident in experimental autoimmune encephalomyelitis (EAE) $[23,26]$. However, it is not known whether ARMD is consistently evident in experimental disease models, leads to a corresponding increase in mitochondrial respiratory chain complex IV activity in demyelinated axons, and how these mitochondrial changes relate to axon degeneration are not known.

Against this background, we used nine experimental disease models and quantified the mitochondrial content, as well as mitochondrial respiratory chain complex IV (COX) activity of demyelinated axons. As complex IV deficiency may be due to the loss of subunits, following mitochondrial DNA mutations, or modification of subunits by reactive oxygen species, we assessed complex IV subunit-I (COX-I) expression in complex IV deficient axonal mitochondria to gain insight into the potential cause of complex IV deficiency. Finally, we correlated complex IV activity within axonal injury in demyelinating lesions. We found ARMD 
to be a consistent feature of all nine animal models. However, COX activity was only increased significantly following lysolecithin. In all experimental disease models, COX-I was intact in COX deficient axonal mitochondria indicating inhibition and post-translational modification, rather than the lack of subunits due to mtDNA deletions and transcriptional changes, as the main cause of COX deficiency in animal models. Correlation of COX deficiency with the extent of axon degeneration suggests a crucial role for COX in meeting the increased energy demand of the acutely demyelinated axon. This study extends our previous characterisation of ARMD by including axonal complex IV activity in multiple animal models and highlighting the need to protect complex IV activity of mitochondria in demyelinated axons for neuroprotection. 


\section{Results}

Increased mitochondrial content within demyelinated axons indicates ARMD in experimental disease models irrespective of the mode of demyelination

We assessed the mitochondrial content of demyelinated axons in nine different disease models at peak clinical disease or, when a clinical phenotype is not yet present, peak demyelination time point (Table 1) and compared it with myelinated axons in controls (Table 2). Mitochondria within demyelinated axons, selected by neurofilament labeling and lack of myelin basic protein (MBP) immunofluorescence, were identified in confocal images based on immunofluorescent labeling of porin, which is a voltage gated anion channel (VDAC) expressed in all mitochondria. (Fig. 1). The mitochondrial content within demyelinated axons was significantly increased in the spinal cord of all EAE, lysolecithin, lipopolysaccharide (LPS) and Theiler's murine encephalomyelitis virus (TMEV) induced models as well as in the corpus callosum in cuprizone mediated demyelination, compared with myelinated axons in controls, indicating the presence of ARMD (Fig. 1 and Table 1). The significant increase in mitochondrial content within demyelinated axons of all models arose from increased mitochondrial size and/or increased mitochondrial number (Table 2). In lysolecithin and cuprizone mediated demyelination and TMEV induced demyelination the average axonal mitochondrial size was significantly greater than in control myelinated axons. Meanwhile, the greater mitochondrial number accounted for the increased mitochondrial content in EAE models. These observations show that ARMD is a consistent phenomenon irrespective of the mode of demyelination.

Increase in axonal mitochondrial content is not always accompanied by a corresponding increase in mitochondrial respiratory chain complex IV activity

To determine whether the increased mitochondrial content within demyelinated axons is reflected at the functional level, we assessed complex IV activity of mitochondria at a single axon level using an established technique involving sequential COX histochemistry and immunofluorescent labeling of axons in snap frozen serial cryosections (Fig. 2) [31]. This sequential technique labels mitochondria with complex IV activity (in brightfield image). Furthermore, this technique identifies mitochondria that lack complex IV activity (in immunofluorescent images) and permits the determination of the subunit status of mitochondria that lack complex IV activity, as previously described [15, 31]. Quantification of complex IV activity within axons revealed a significantly greater area of the demyelinated axons occupied by complex IV active mitochondria in lysolecithin-induced lesions (Figure 2 
and Table 3). In these focal lesions, complex IV active mitochondria with elongated morphology were prevalent within demyelinated axons (Fig. 2). The remaining models did not show a significant increase in complex IV activity within demyelinated axons. Although complex IV activity had a tendency to increase within demyelinated axons in cuprizone and TMEV models the difference was not statistically significant.

Mitochondrial respiratory chain complex IV subunit-I is preserved in complex IV deficient axonal mitochondria in all experimental disease models

In axonal mitochondria that lack complex IV activity, the extent of complex IV subunit-I (COXI) labeling was similar in demyelinated axons when compared with myelinated axons, suggesting that that the lack of complex IV activity is not caused by the loss of complex IV subunit-I. To confirm that the complex IV subunit is intact in demyelinated axons, we immunofluorescently co-labeled mitochondria and the subunit in serial sections and found a significant increase in complex IV subunit-I within demyelinated axons compared with myelinated axons (Table 3). The regions with inflammatory infiltrates in EAE showed a diffuse loss of complex IV activity and upregulation of iNOS [13]. These findings suggest that complex IV activity is acutely inhibited and/or complex IV subunits are post-translationally modified by the inflammatory response in a proportion of axonal mitochondria which had moved to the axon through ARMD as well as those already present in the axon before demyelination [21].

Mitochondrial complex IV activity in demyelinated axons inversely correlates with axonal injury in experimental disease models

We assessed the relationship between axonal mitochondrial parameters (content and complex IV activity) and axonal damage as indicated by the density of APP and synaptophysin positive elements (Fig. 3). We did not detect a significant correlation between axonal mitochondrial content and axonal damage. However, at the level of complex IV activity, there was a significant inverse correlation between the mean complex IV activity within demyelinated axons and the density of synaptophysin positive elements (Fig. 3). APP-positive elements also showed a significant inverse relationship with axonal complex IV activity (Fig. 3). The inverse correlation between axonal complex IV activity and axonal damage indicates the importance of preserving complex IV activity in acutely demyelinated axons. 


\section{Discussion}

We recently reported a homeostatic mechanism in neurons, termed ARMD, where mitochondria move from the cell body to the axon upon lysolecithin induced focal demyelination [13]. In the present study we show that ARMD is a consistent feature of demyelinated axons in nine experimental disease models. In contrast, complex IV activity was not consistently increased in demyelinated axons, particularly in the EAE models studied, and axonal complex IV activity inversely correlated with axonal injury. These findings identify preservation of complex IV activity in axonal mitochondria that have responded to demyelination as an important strategy for neuroprotection in inflammatory demyelination.

Previous studies have identified increased mitochondrial content in demyelinated axons in ethidium bromide (EB) lesions, demyelinated cat optic nerve and Theiler's murine encephalomyelitis virus (TMEV) as well as in MS [15, 17, 24, 32]. In this study we show that the axonal mitochondrial content consistently increases, irrespective of the mode of demyelination, due to increased size and/or number of axonal mitochondria. The lack of increased mitochondrial size in inflammatory demyelination may be due to mitochondrial fragmentation, as previously reported in EAE, as well as decreased fusion of mitochondria in demyelinated axons. Whether mitochondrial fusion occurs within demyelinated axons in EAE, before mitochondrial fragmentation, needs to be investigated using live imaging techniques. While the increased axonal mitochondrial content has been proposed as a pathogenic mechanism, a study that prevented the increase of mitochondrial content in demyelinated axons by disrupting mitochondrial docking established that ARMD is a homeostatic and protective mechanism [19]. Furthermore, recent studies that enhanced ARMD, by overexpressing PGC1a in neurons as well as pharmacologically targeting PGC1a to increase mitochondrial biogenesis, showed protection of acutely demyelinated axons in EAE and lysolecithin-induced lesions [13, 22]. Our current findings in nine experimental models robustly establish that ARMD is a consistent compensatory response to demyelination.

Whether the increased mitochondrial content of demyelinated axons is reflected at the level of mitochondrial complex IV activity had not been studied in experimental disease models. In this study, we show that the extent of complex IV activity of demyelinated axons is dependent on the mode of experimental demyelination (Fig. 4). The finding that lysolecithin induced lesions, showing a significant increase of complex IV activity in demyelinated axons, is comparable to findings in dysmyelinated axons in shiverer mice, where inflammation is 
minimal [3]. In contrast, autoimmune inflammation did not lead to a significant increase of axonal complex IV activity despite the increase of axonal mitochondrial content (Fig. 4). The loss of complex IV activity in axonal mitochondria that have responded to demyelination in EAE may be due to a number of reasons. Firstly, a previous study has shown that modification of complex IV subunits occurs in EAE, although this was not localised to particular cellullar structures [21]. Secondly, an excess of nitric oxide which is known to compete with oxygen and inhibit complex IV is evidenced by the increase in iNOS in EAE $[13,25]$ Nitric oxide is also implicated in axon degeneration due to the correlation between iNOS expression and acute axonal injury [1]. In TMEV induced demyelination, the relative sparing of complex IV activity at 41 days is likely to be due to weak expression of iNOS and reactive oxygen species at this stage $[11,20]$. Thirdly, reactive oxygen species damage axonal mitochondria and disrupt axonal mitochondrial transport in $\operatorname{EAE}[18,26]$. The differential complex IV activity within axons in toxic demyelination and inflammatory demyelination is consistent with inflammation related complex IV deficiency in demyelinating models.

Our findings of intact COX-I indicate that the lack of complex IV acitivity in experimentally demyelinated axons in EAE is due to inhibition or post-translational modification of complex IV subunits rather than due to mitochondrial DNA (mtDNA) mutations or loss of transcripts. These observations in EAE are supported by previous studies of transcripts of mitochondrial respiratory chain subunits and mtDNA, both of which were unaltered in experimental models $[4,13]$. While experimental disease models with inflammatory demyelination represent complex IV deficiency in axons, at a mechanistic level these models only represent inflammation induced complex IV deficiency, rather than the irreversible complex IV deficiency due to mitochondrial DNA mutations that are found in MS $[5,13]$.

Complex IV activity is important for axonal health in non-demyelinating disorders and likely to be even more relevant in demyelinating disorders as a result of the increased energy demand of demyelinated axons. For the first time, we show an inverse relationship between complex IV activity in demyelinated axons and extent of axon degeneration in experimental disease models, suggesting the importance of preserving mitochondrial respiratory chain function in demyelinated axons. We recently showed that enhancing ARMD in complex IV deficient neurons can protect demyelinated axons in COX10Adv mice [13]. This indicates that complex IV deficiency in axons plays a role in the axon degeneration through the loss of function or lack of energy rather than due to a toxic gain of function. Unlike in COX10Adv mice, where the complex IV deficiency is irreversible, complex IV deficiency due to inhibition by nitric oxide and 
modification of subunits by reactive oxygen species, as evidenced in certain experimental disease models, is potentially reversible. This reversibility stems from the ability of neurons in experimental disease models to generate healthy mitochondria as they do not show mtDNA mutations or loss of nuclear DNA encoded transcripts $[4,13]$. In these experimental disease models, newly generated mitochondria and their movement from the cell body to the axon may replace damaged mitochondria and restore axonal energy production. In MS, complex IV deficiency of axons is caused by multiple mechanisms, including nitric oxide mediated inhibition of complex IV, inflammation related direct damage to complex IV as well as the chronic nature of oxidative injury leading to mtDNA deletions. The co-occurence of potentially reversible complex IV deficiency due to inflammation induced mitochondrial damage with the irreversible complex IV deficiency due to mitochondrial DNA mutations in MS offers therapeutic potential $[5,15]$.

In summary, we show that ARMD is a consistent feature of a wide range of experimental disease models and highlight the importance of preserving axonal complex IV activity in inflammatory demyelinating disorders. Our findings suggest that enhancing ARMD as a neuroprotective strategy for MS may be further optimised by limiting damage to complex IV, through combinatorial therapies including immunomodulation. 
Table 1. Features of the disease models

\begin{tabular}{|c|c|c|c|}
\hline Model (co-author) & $\begin{array}{l}\text { species } \\
\text { (strain) }\end{array}$ & $\begin{array}{l}\text { time points for analysis } \\
\text { in days }\end{array}$ & $\begin{array}{l}n=\text { (brain, } \\
\text { spinal } \\
\text { cord) }\end{array}$ \\
\hline $\begin{array}{l}\text { focal demyelinating dorsal } \\
\text { funiculus lesion: } \\
\text { LPC }(1 \%)[30]\end{array}$ & $\begin{array}{l}\text { Mouse } \\
\text { (C57BL/6) }\end{array}$ & $5^{*}$ & 0,6 \\
\hline LPS (200ng)[8] & $\begin{array}{l}\text { Rat } \\
\text { (Sprague- } \\
\text { Dawley) }\end{array}$ & $7^{*}$ & 0,6 \\
\hline $\begin{array}{l}\text { Cuprizone-mediated } \\
\text { demyelination of the brain } \\
{[10]}\end{array}$ & $\begin{array}{l}\text { Mouse } \\
\text { (C57BL/6) }\end{array}$ & $42^{*}$ & 6,0 \\
\hline $\begin{array}{l}\text { TMEV-induced inflammatory } \\
\text { demyelination [24] }\end{array}$ & $\begin{array}{l}\text { Mouse } \\
\text { (SJL/J) }\end{array}$ & 41 (demyelinating) & 3,3 \\
\hline $\begin{array}{l}\text { T-reg depleted active EAE } \\
\text { with } \mathrm{MOG}_{35-55}[16]\end{array}$ & $\begin{array}{l}\text { Mouse } \\
\text { (C57BL/6) }\end{array}$ & 13 (acute) $^{*}$ & 0,10 \\
\hline $\begin{array}{l}\text { humanized TCR transgenic } \\
\text { with spontaneous EAE [7] }\end{array}$ & $\begin{array}{l}\text { Mouse } \\
\text { (C57BL/6) }\end{array}$ & $\begin{array}{l}120-150^{*}, \quad \text { (clinical } \\
\text { score } 1-2 \text { and }>3 \text { ) }\end{array}$ & 3,3 \\
\hline $\begin{array}{ll}\text { chronic EAE } & \text { with } \\
\text { subcutaneous spinal } & \text { cord } \\
\text { homogenate [2] } & \end{array}$ & $\begin{array}{l}\text { Mouse } \\
\text { (Biozzi ABH) }\end{array}$ & 18 (acute) $^{*}$ & 3,3 \\
\hline acute EAE with rMOG [6] & $\begin{array}{l}\text { Rat } \\
\text { (Dark Agouti, } \\
\text { Harlan) }\end{array}$ & $14^{*}$ & 3,3 \\
\hline EAE with $\mathrm{rMOG}_{34-56}$ [12] & $\begin{array}{l}\text { Marmoset } \\
\text { (Callithrix } \\
\text { jacchus) }\end{array}$ & $\begin{array}{l}11 \text { days }^{*}, \text { on average, } \\
\text { post EAE score of } 2.5\end{array}$ & 9,5 \\
\hline
\end{tabular}


*indicates peak clinical disease or peak demyelination time point for the analysis of axonal mitochondrial parameters. All the time-points stated above were included in the detection for respiratory-deficient cells. EAE: experimental autoimmune encephalomyelitis. LPC: Iysolecithin. LPS: lipopolysaccharide. MOG: myelin oligodendrocyte glycoprotein. TCR: T-cell receptor. TMEV: Theiler's murine encephalomyelitis virus. $n=$ number of animals used for brain and spinal cord analysis. Equal numbers of age-matched controls were used, except for marmoset EAE, where 4 age-matched naïve controls were used. 
Table 2. Changes in axonal mitochondrial content, size and number following demyelination in models and MS

\begin{tabular}{|c|c|c|c|}
\hline model & $\begin{array}{l}\text { mitochondrial } \\
\text { content (\% of } \\
\text { axon area) }\end{array}$ & $\begin{array}{l}\text { mitochondrial } \\
\text { size }\left(\mu m^{2}\right)\end{array}$ & $\begin{array}{l}\text { mitochondrial } \\
\text { number } / 10^{3} \mu \mathrm{m}^{2} \\
\text { of axon area }\end{array}$ \\
\hline \multirow[t]{2}{*}{ focal LPC } & $28.78 \pm 9.98^{* * *}$ & $5.28 \pm 1.77^{* *}$ & $42.07 \pm 19.39$ \\
\hline & $15.02 \pm 3.57$ & $2.93 \pm 1.56$ & $32.28 \pm 19.36$ \\
\hline \multirow[t]{2}{*}{ focal LPS } & $23.88 \pm 12.98^{* * *}$ & $4.55 \pm 2.24$ & $56.40 \pm 25.57^{*}$ \\
\hline & $13.17 \pm 3.73$ & $4.58 \pm 1.30$ & $40.11 \pm 14.02$ \\
\hline \multirow[t]{2}{*}{ cuprizone-mediated } & $23.01 \pm 4.99^{* * *}$ & $4.75 \pm 1.71^{* *}$ & $68.81 \pm 12.21^{* * *}$ \\
\hline & $15.02 \pm 3.57$ & $2.92 \pm 1.03$ & $47.66 \pm 7.71$ \\
\hline \multirow[t]{2}{*}{ TMEV-induced } & $20.39 \pm 2.88^{* *}$ & $6.61 \pm 2.29^{* *}$ & $34.71 \pm 8.62$ \\
\hline & $15.17 \pm 2.04$ & $4.56 \pm 1.29$ & $33.45 \pm 10.92$ \\
\hline \multirow[t]{2}{*}{ TCR tg EAE } & $16.76 \pm 7.30^{*}$ & $5.01 \pm 1.09$ & $40.76 \pm 20.51^{*}$ \\
\hline & $10.15 \pm 4.00$ & $4.26 \pm 1.21$ & $23.30 \pm 13.13$ \\
\hline \multirow[t]{2}{*}{ EAE Biozzi ABH } & $20.81 \pm 7.42^{* * *}$ & $4.93 \pm 1.29$ & $43.04 \pm 14.74^{* *}$ \\
\hline & $12.79 \pm 3.22$ & $4.69 \pm 1.24$ & $27.75 \pm 5.16$ \\
\hline \multirow[t]{2}{*}{ T-reg depleted EAE } & $23.18 \pm 9.79^{* * *}$ & $2.57 \pm 0.97$ & $53.46 \pm 14.77$ \\
\hline & $15.38 \pm 4.88$ & $1.98 \pm 1.42$ & $32.04 \pm 16.96$ \\
\hline \multirow[t]{2}{*}{ EAE rat } & $25.74 \pm 7.72^{* * *}$ & $5.10 \pm 2.62$ & $54.90 \pm 13.78^{* * *}$ \\
\hline & $13.37 \pm 3.91$ & $3.78 \pm 1.24$ & $37.15 \pm 10.39$ \\
\hline \multirow[t]{2}{*}{ EAE marmoset } & $19.51 \pm 5.92^{*}$ & $3.08 \pm 0.64$ & $64.93 \pm 21.44$ \\
\hline & $14.92 \pm 8.33$ & $3.31 \pm 1.93$ & $53.18 \pm 12.49$ \\
\hline \multirow{2}{*}{$\begin{array}{l}\text { Progressive } \\
\text { (Zambonin et al., } \\
2011)\end{array}$} & $19.61 \pm 5.67^{* *}$ & $15.67 \pm 3.68^{* *}$ & $49.21 \pm 11.93$ \\
\hline & $6.26 \pm 1.73$ & $5.22 \pm 1.92$ & $33.60 \pm 9.67$ \\
\hline
\end{tabular}

Table 2. Mitochondrial content (column two) is expressed as a percentage of axonal area occupied by porin-labelled elements. Mitochondrial size (column three) and mitochondrial number (column four) are based on the area and number, respectively, of porin-labelled elements within axons in confocal images. Shaded rows indicate mean values for myelinated axons from controls and unshaded rows mean indicate values for demyelinated axons. EAE: experimental autoimmune encephalomyelitis. LPC: Iysolecithin. LPS: lipopolysaccharide. TCR tg: T-cell receptor transgenic. TMEV: Theiler's murine encephalomyelitis virus. Values indicate mean \pm standard deviation. ${ }^{*} p<0.05,{ }^{* *} p<0.01$ and ${ }^{* * *} p<0.001$. 
Table 3. Complex IV activity and complex IV subunit-I relative to axonal area and complex IV subunit-I relative complex II subunit labeled area in myelinated axons and demyelinated axons

\begin{tabular}{|c|c|c|c|}
\hline model & $\begin{array}{l}\text { complex IV activity } \\
\text { in all axonal } \\
\text { mitochondria ( } \% \text { of } \\
\text { axon area) }\end{array}$ & $\begin{array}{l}\text { complex IV subunit- } \\
\text { I in complex IV- } \\
\text { deficient } \\
\text { mitochondria (\% of } \\
\text { complex II } 70 \mathrm{KDa} \\
\text { area) }\end{array}$ & $\begin{array}{l}\text { complex IV subunit- } \\
\text { I in all axonal } \\
\text { mitochondria ( } \% \text { of } \\
\text { axonal area) }\end{array}$ \\
\hline \multirow[t]{2}{*}{ Focal LPC } & $12.81 \pm 3.69^{* *}$ & $85.49 \pm 16.96$ & $24.15 \pm 8.37^{* *}$ \\
\hline & $7.44 \pm 4.57$ & $62.22 \pm 11.59$ & $10.25 \pm 2.43$ \\
\hline \multirow[t]{2}{*}{ Focal LPS } & $6.08 \pm 2.55$ & $63.16 \pm 21.29$ & $15.51 \pm 8.43^{* *}$ \\
\hline & $5.84 \pm 1.78$ & $69.13 \pm 14.51$ & $8.49 \pm 2.48$ \\
\hline \multirow{2}{*}{$\begin{array}{l}\text { Cuprizone- } \\
\text { mediated }\end{array}$} & $10.50 \pm 6.55$ & $68.73 \pm 14.24$ & $17.30 \pm 3.75^{\star *}$ \\
\hline & $6.83 \pm 1.55$ & $61.34 \pm 11.98$ & $11.08 \pm 2.25$ \\
\hline \multirow{2}{*}{$\begin{array}{l}\text { TMEV- } \\
\text { induced }\end{array}$} & $9.42 \pm 6.37$ & $58.41 \pm 14.14$ & $15.89 \pm 2.91^{* *}$ \\
\hline & $4.98 \pm 1.19$ & $62.88 \pm 10.87$ & $10.89 \pm 2.44$ \\
\hline \multirow{2}{*}{$\begin{array}{l}\text { EAE, } \quad \text { TCR } \\
\operatorname{tg}\end{array}$} & $4.28 \pm 2.48$ & $73.46 \pm 15.32$ & $11.61 \pm 5.31^{*}$ \\
\hline & $4.59 \pm 1.38$ & $67.25 \pm 11.14$ & $7.72 \pm 2.87$ \\
\hline \multirow{2}{*}{$\begin{array}{l}\text { EAE, Biozzi } \\
A B H\end{array}$} & $3.69 \pm 2.15$ & $62.36 \pm 21.20$ & $15.16 \pm 5.28^{* *}$ \\
\hline & $5.07 \pm 2.16$ & $67.54 \pm 10.26$ & $8.76 \pm 2.09$ \\
\hline \multirow{2}{*}{$\begin{array}{l}\text { EAE, T-reg } \\
\text { depleted }\end{array}$} & $2.18 \pm 1.15$ & $64.73 \pm 18.56$ & $14.24 \pm 4.96^{*}$ \\
\hline & $6.10 \pm 1.11$ & $71.96 \pm 11.90$ & $9.32 \pm 2.23$ \\
\hline \multirow[t]{2}{*}{$\mathrm{EAE}$, rat } & $4.36 \pm 1.90$ & $64.78 \pm 20.50$ & $17.59 \pm 5.28^{* *}$ \\
\hline & $5.57 \pm 2.50$ & $67.83 \pm 12.17$ & $8.12 \pm 2.38$ \\
\hline \multirow{2}{*}{$\begin{array}{l}\mathrm{EAE}, \\
\text { marmoset }\end{array}$} & $5.01 \pm 1.39$ & $66.49 \pm 22.95$ & $14.71 \pm 4.46^{*}$ \\
\hline & $5.74 \pm 1.98$ & $70.76 \pm 18.49$ & $10.13 \pm 5.66$ \\
\hline \multirow[t]{2}{*}{$\begin{array}{l}\text { Progressive } \\
\text { MS }\end{array}$} & $\begin{array}{l}7.37 \quad \pm \quad 4.98^{* *} \\
\text { (Mahad et } \quad \text { al., } \\
2009 \text { ) }\end{array}$ & $22.82 \pm 21.34^{* *}$ & $10.10 \pm 3.89^{* *}$ \\
\hline & $2.23 \pm 1.62$ & $51.89 \pm 22.86$ & $4.91 \pm 3.05$ \\
\hline
\end{tabular}


Table 3. Mitochondrial respiratory chain complex IV active mitochondria in axons (column two) are assessed as a percentage of axonal area occupied by these complex IV active mitochondria. Complex IV subunit-I is assessed in all axonal mitochondria as the percentage area of the subunit present within the axons (column three) in triple labeled images. When axonal mitochondria that lack complex IV activity are detected using complex II 70kDa labelled elements within axons by the sequential COX histochemistry and triple labeling technique, the percentage area of complex IV subunit-I labeling in the mitochondria are not significantly different between myelinated axons in controls and demyelinated axons in all the disease models (last columns). Shaded rows indicate values for myelinated axons and unshaded rows indicate values for demyelinated axons. EAE: experimental autoimmune encephalomyelitis. LPC: Iysolecithin. LPS: lipopolysaccharide. TCR tg: T-cell receptor transgenic. TMEV: Theiler's murine encephalomyelitis virus. Values indicate mean \pm standard deviation. ${ }^{*} p<0.01$ and ${ }^{* *} p<0.001$. 
Figure 1

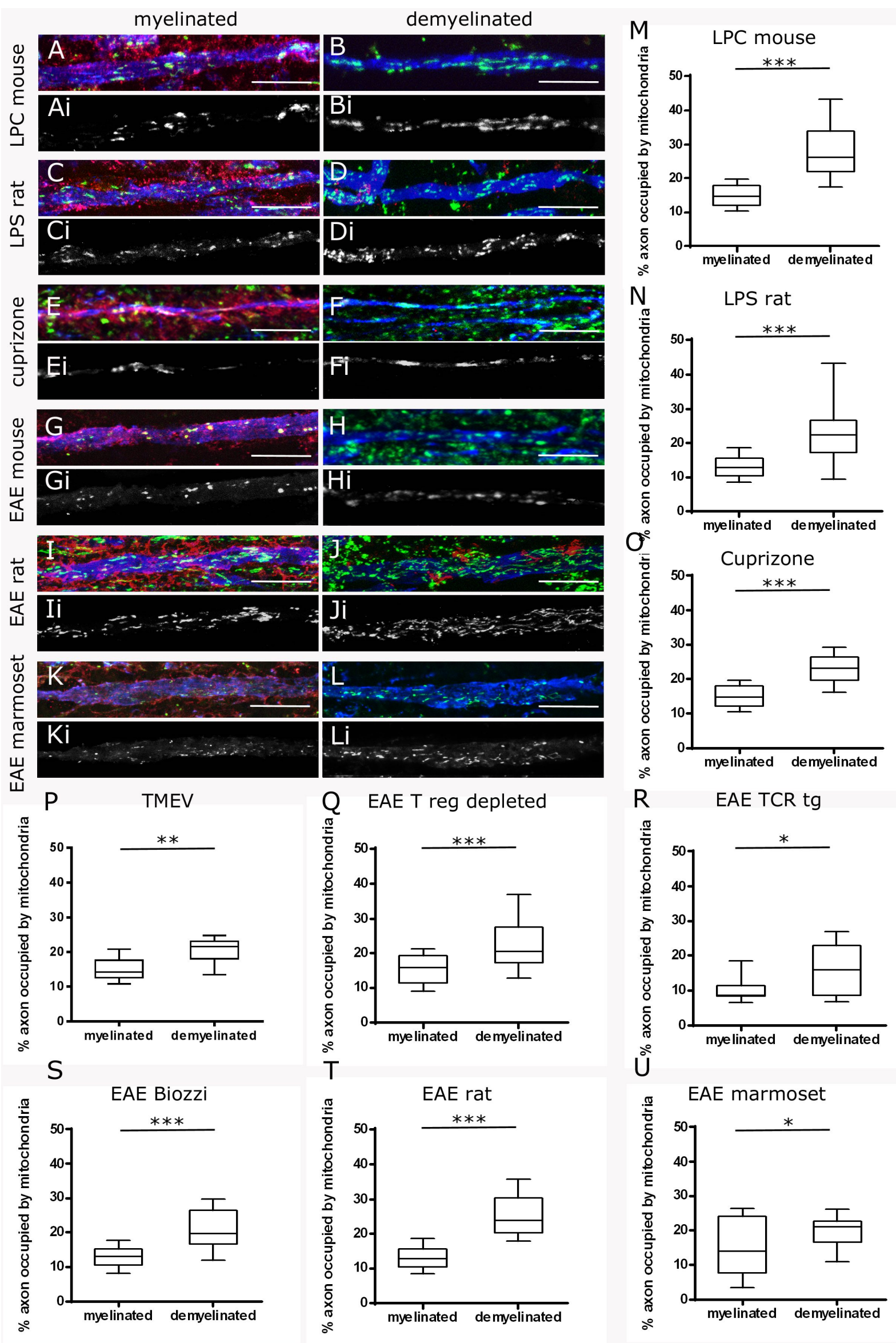


Figure 1. Axonal mitochondrial content consistently increases upon experimental demyelination. Compared with myelinated axons from controls, in triple labeled immunofluorescent confocal images (column to the left with MBP in red, neurofilament-H in blue and porin in green), mitochondria are more prevalent in demyelinated axons (column to the right) from all the models. The grey scale images (Ai-Li) show porin-positive elements within axons from the corresponding triple labeled colour images (A-L). The quantitation of axonal mitochondrial content shows a significant increase in the lysolecithin-induced focal lesions (LPC, A-B and M), lipopolysaccharide-induced focal lesions (LPS, C-D and N), cuprizone model (E-F and O), Theiler's murine encephalomyelitis virus (TMEV) model $(P)$ as well as experimental autoimmune encephalitis (EAE, G-L and Q-U) in mice (C57BL6, SJL/J and Biozzi $A B H$ ), rat (Dark agouti) and marmoset species (the area of porin-positive elements as a percentage of axon area). 20 axons per region were randomly selected from each animal for quantitation. The box plots indicate the median, inter-quartile range (25\%-75\%) and 90\% confidence interval. ${ }^{*} p<0.05,{ }^{* *} p<0.01$ and ${ }^{* * *} p<0.01$. Scale bar indicates $10 \mu \mathrm{m}$. 
Figure 2

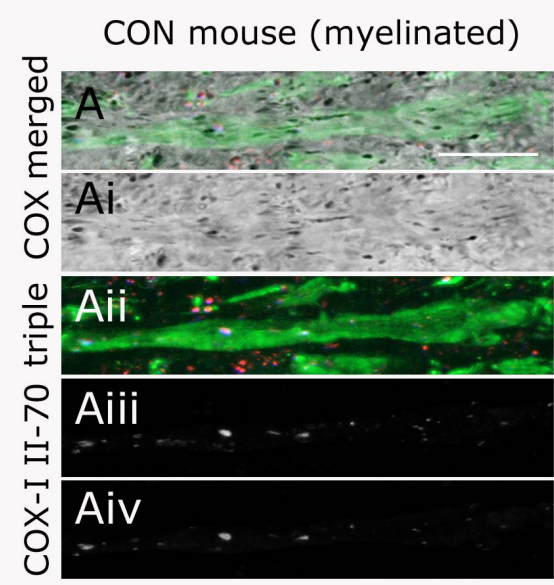

LPC mouse (demyelinated)

LPS rat (demyelinated)
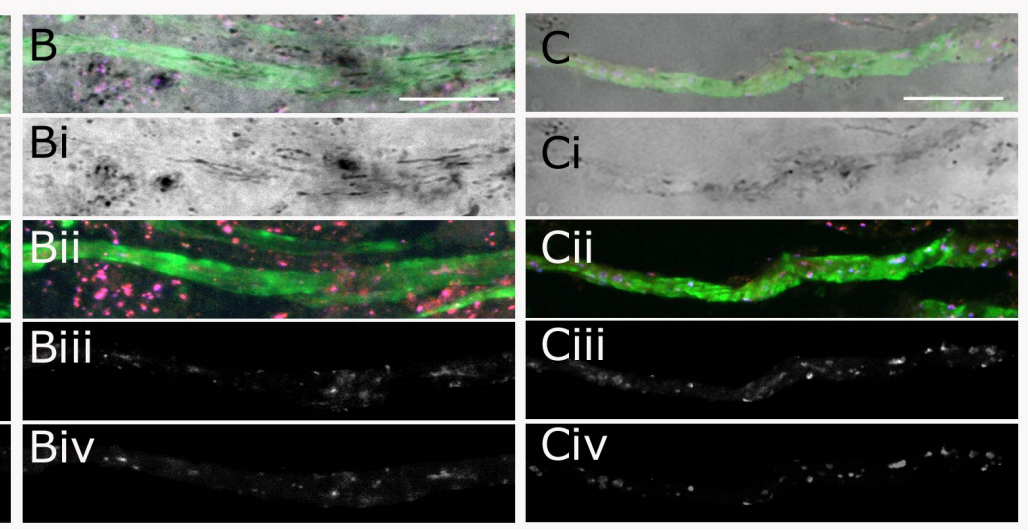

cuprizone (demyelinated)

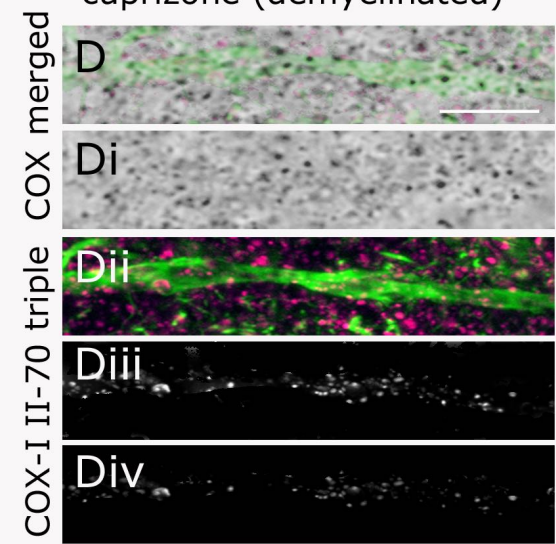

TMEV (demyelinated)

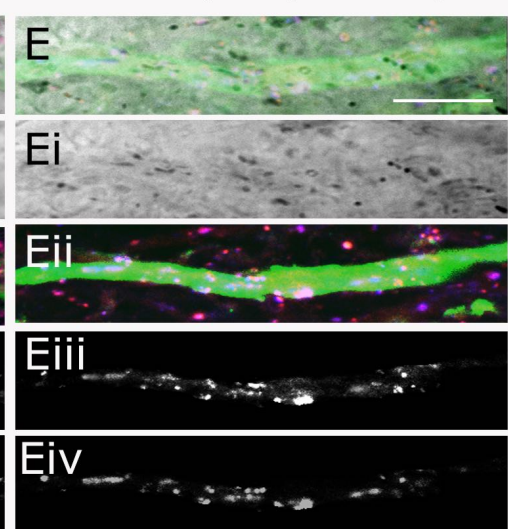

EAE TCR tg (demyelinated)

EAE rat (demyelinated)
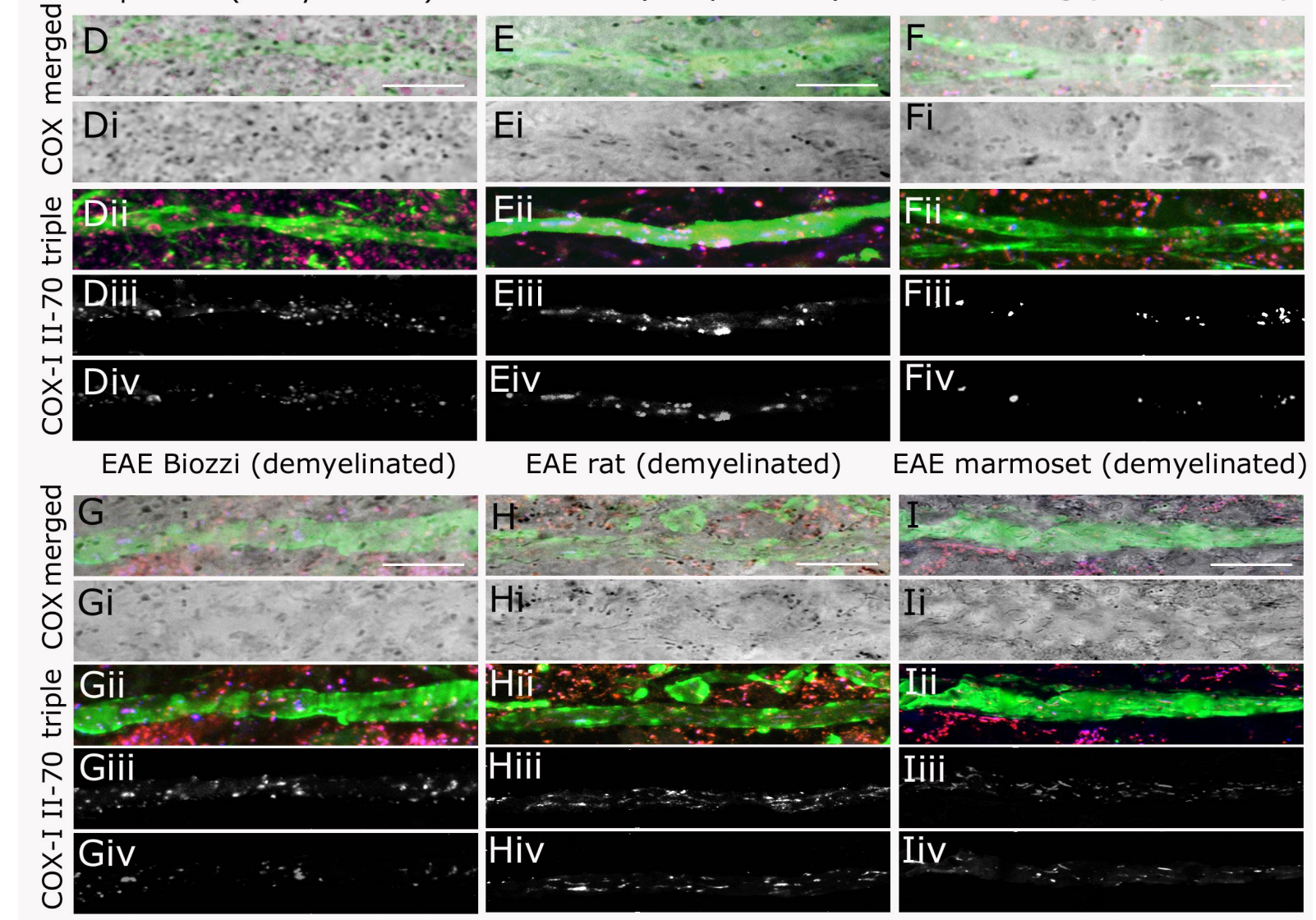

EAE marmoset (demyelinated)
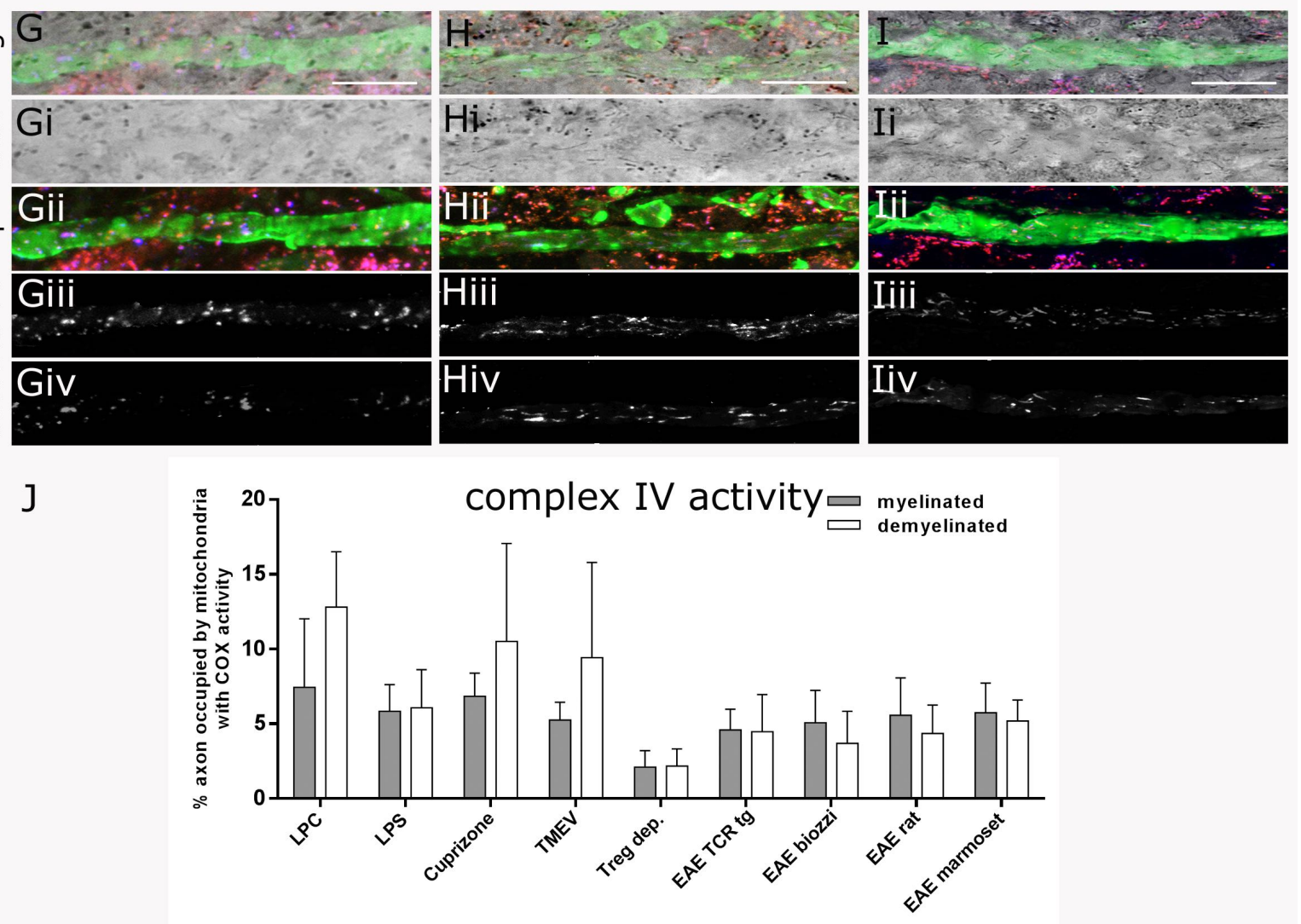
Figure 2. Complex IV activity within axons and the detection of complex IV subunit-I relative to complex II 70kDa in complex IV-deficient axonal mitochondria.

Complex IV activity can be localized to the axon by the sequential complex IV histochemistry (bright field images, Ai-li) and triple immunofluorescent labeling (Aii-lii) of neurofilament (green), complex II 70kDa subunit (red) and complex IV subunit-I (blue) and then by merging the bright field image with triple labeled immunofluorescent image (A-I). This sequential technique immunofluorescently labels the complex IV-deficient mitochondria (labeled with complex II 70kDa, Aiii-liii) and their mitochondrial respiratory chain complex subunits (Aiv-liv), as previously described [31]. The grey scale immunofluorescent images of axonal complex II 70kDa (Aiii-liii) and complex IV subunit-I (Aiv-liv) are generated by splitting the corresponding triple labeled colour image (Aii-lii) and clearing the non-axonal mitochondria. As reported previously, the mitochondria with complex IV activity, evident in the bright field images, are not immunofluorescently labelled [31]. Following lysolecithin-induced (LPC) demyelination (panel $\mathrm{B})$, there are numerous axonal mitochondria with complex IV activity and elongated morphology ( $\mathrm{B}$ and $\mathrm{Bi}$ ) compared with myelinated axons from controls ( $\mathrm{A}$ and $\mathrm{Ai}$ ). In contrast, mitochondria with complex IV activity in demyelinated axons are less numerous and rounded or less elongated in all other models [lipopolysaccharide-induced (LPS) lesions (C-Ci), cuprizone model (D-Di), TMEV model and experimental autoimmune encephalitis (EAE) in mouse, rat and marmoset]. The quantitation of complex IV activity within axons shows a significant increase following LPC-induced focal demyelination (J). 20 axons per region were randomly selected from each animal for quantitation. The bar charts indicate the mean plus standard deviation. ${ }^{*} p<0.001$. Scale bar indicates $10 \mu \mathrm{m}$. 
Figure 3

A

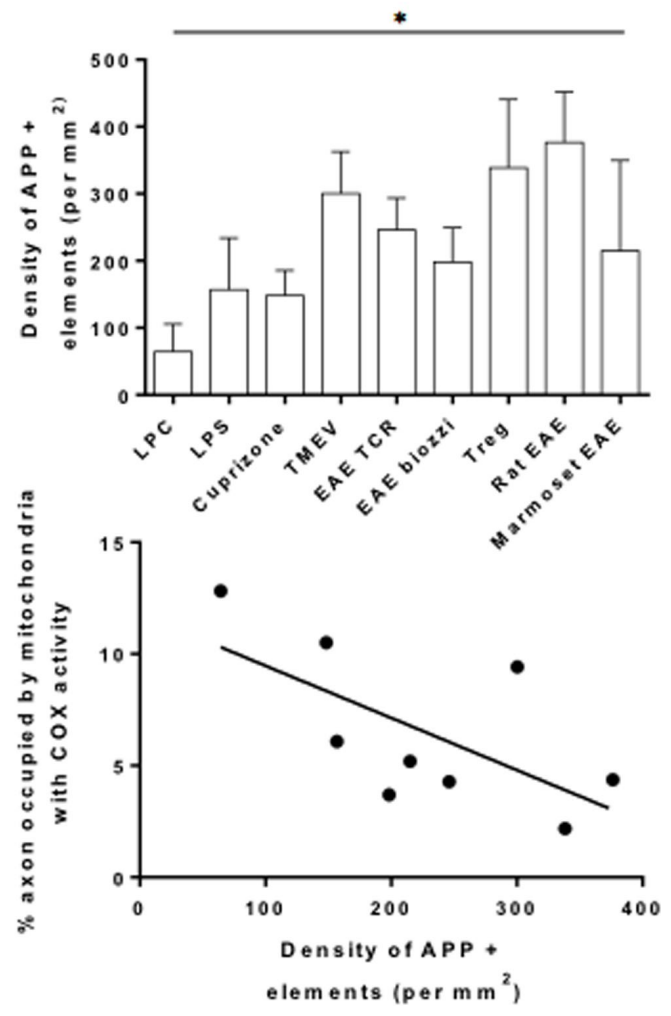

C

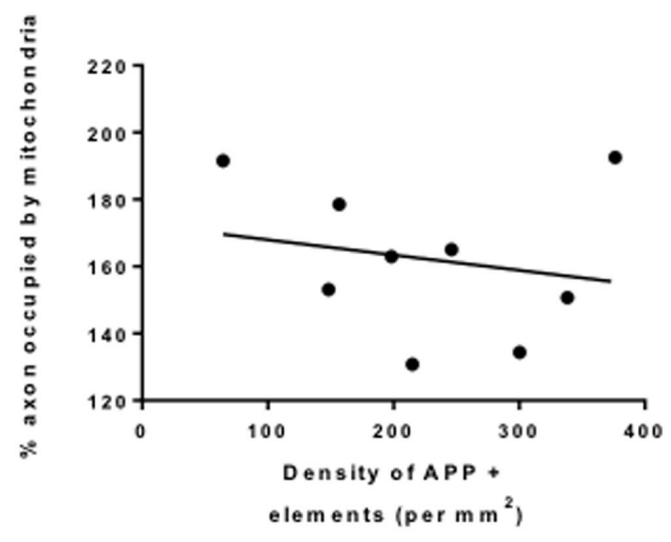

$\mathrm{Ai}$

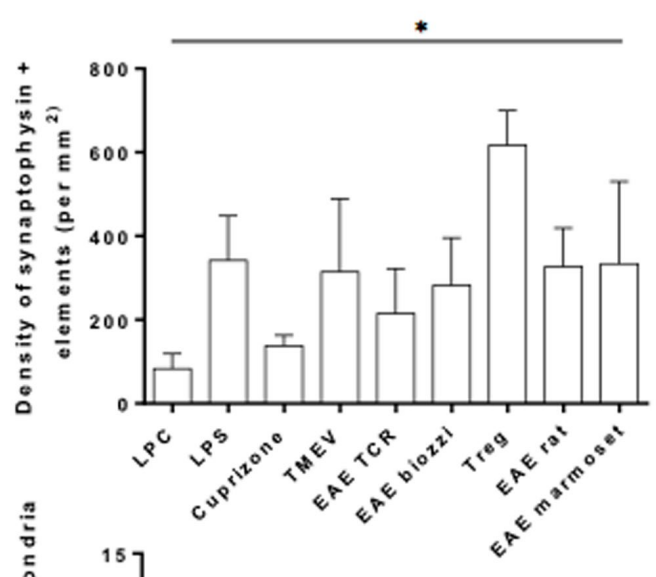

$\mathrm{Bi}$

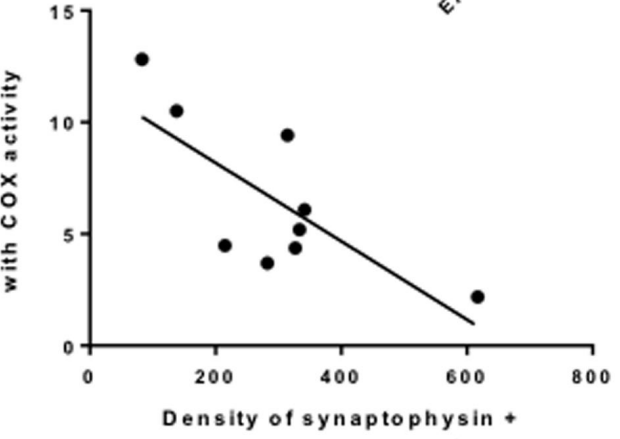

$\mathrm{Ci}$

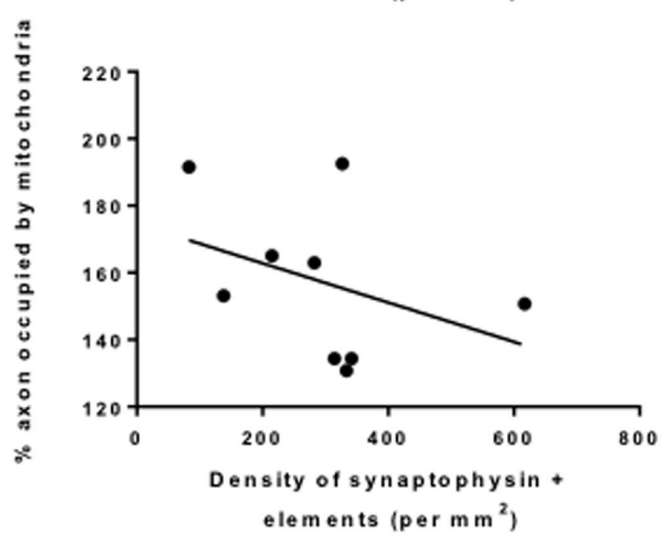

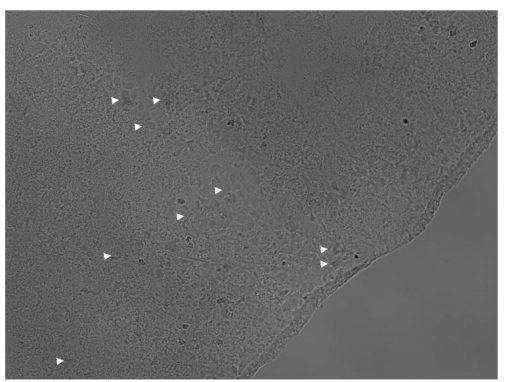
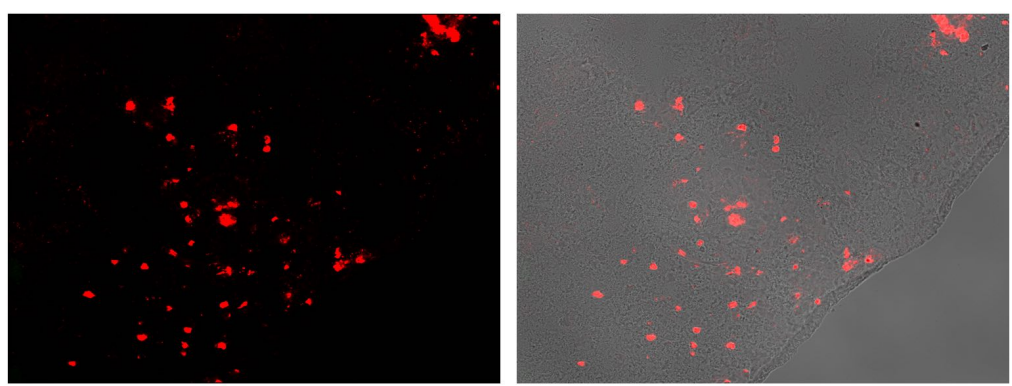

Figure 3. The association between complex IV activity within demyelinated axons and extent of axonal injury. The density of axonal injury, judged by amyloid precursor protein (APP, A) and synaptophysin $(\mathrm{Ai})$ labelling, varies considerably between the disease models (ANOVA 
$\mathrm{p}<0.001)$. There is a significant inverse correlation between complex IV activity within demyelinated axons and axon degeneration, judged by the density of APP $\left(B, r^{2}=0.421\right.$, $\mathrm{p}=0.048)$ as well as synaptophysin $\left(\mathrm{Bi}, \mathrm{r}^{2}=0.561, \mathrm{p}=0.020\right)$ labelling. In contrast, a significant correlation is not found between mitochondrial content in demyelinated axons and the density of $\operatorname{APP}\left(C, r^{2}=0.040, p=0.604\right)$ and synaptophysin $\left(C i, r^{2}=0.147, p=0.308\right)$ labeling. Sequential COX histochemistry and immunofluorescent labelling of APP shows that a subset of APP and synaptophysin labelled structures contains mitochondria with complex IV activity in all nine disease models $(D$, synaptophysin positive structures lacking complex IV activity are shown in T-reg depleted EAE lesion, arrowheads). The bar charts indicate the mean plus standard deviation. 
Figure 4

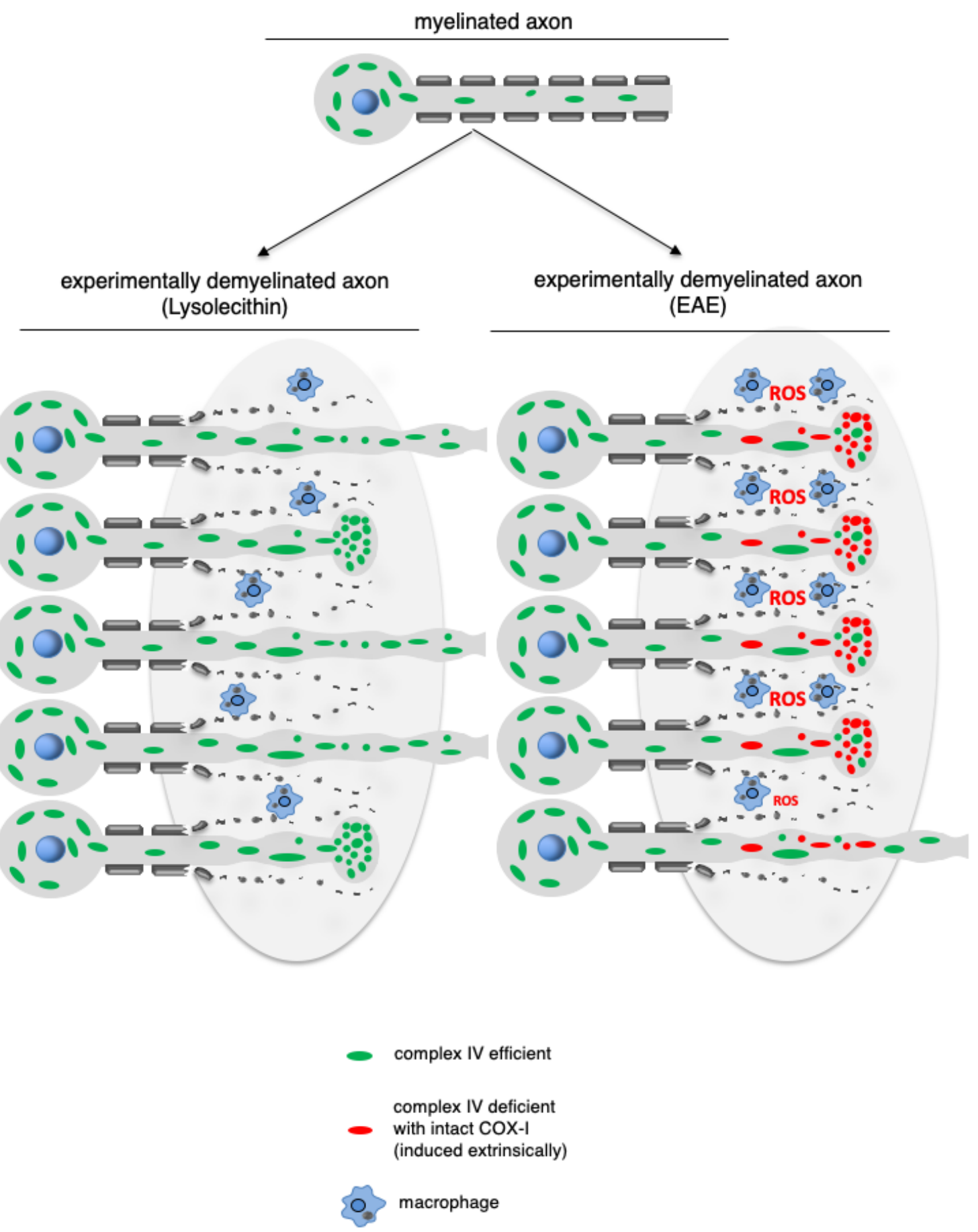

Figure 4. The role of complex IV in ARMD. Compared with myelinated axons, demyelinated axons consistently show increased mitochondrial content irrespective of the mode of 
experimental demyelination. At the level of complex IV activity (green mitochondria) lysolecithin induced lesions show a significant increase compared with myelinated axons, reflecting the increased axonal mitochondrial content. In contrast, the increased mitochondrial content of demyelinated axons in EAE is not accompanied by a corresponding increase in complex IV activity (red mitochondria). The lack of complex IV activity in demyelinated axons is associated with a substantially greater extent of axon injury. Intact COX-I in complex IV deficient axonal mitochondria suggests that the complex IV deficiency due to inhibition by nitric oxide and subunit modification by reactive oxygen species (ROS) is potentially reversible through mitochondrial biogenesis and subsequent replacement with healthy mitochondria. 
References

1. Aboul-Enein F, Weiser P, Höftberger R, Lassmann H, Bradl M (2006) Transient axonal injury in the absence of demyelination: a correlate of clinical disease in acute experimental autoimmune encephalomyelitis. Acta Neuropathol 111:539-547. doi: 10.1007/s00401-006-0047-y

2. Al-Izki S, Pryce G, O’Neill JK, Butter C, Giovannoni G, Amor S, Baker D (2012) Practical guide to the induction of relapsing progressive experimental autoimmune encephalomyelitis in the Biozzi ABH mouse. Mult Scler Relat Disord 1:29-38. doi: 10.1016/j.msard.2011.09.001

3. Andrews H, White K, Thomson C, Edgar J, Bates D, Griffiths I, Turnbull D, Nichols P (2006) Increased axonal mitochondrial activity as an adaptation to myelin deficiency in the Shiverer mouse. J Neurosci Res 83:1533-1539. doi: 10.1002/jnr.20842

4. Broadwater L, Pandit A, Clements R, Azzam S, Vadnal J, Sulak M, Yong VW, Freeman EJ, Gregory RB, McDonough J (2011) Analysis of the mitochondrial proteome in multiple sclerosis cortex. Biochim Biophys Acta 1812:630-641. doi: 10.1016/j.bbadis.2011.01.012

5. Campbell GR, Ziabreva I, Reeve AK, Krishnan KJ, Reynolds R, Howell O, Lassmann H, Turnbull DM, Mahad DJ (2011) Mitochondrial DNA deletions and neurodegeneration in multiple sclerosis. Ann Neurol 69:481-492. doi: 10.1002/ana.22109

6. Davies AL, Desai RA, Bloomfield PS, Mclntosh PR, Chapple KJ, Linington C, Fairless R, Diem R, Kasti M, Murphy MP, Smith KJ (2013) Neurological deficits caused by tissue hypoxia in neuroinflammatory disease. Ann Neurol 74:815-825. doi: 10.1002/ana.24006

7. Ellmerich S, Takacs K, Mycko M, Waldner H, Wahid F, Boyton RJ, Smith PA, Amor S, Baker D, Hafler DA, Kuchroo VK, Altmann DM (2004) Disease-related epitope spread in a humanized T cell receptor transgenic model of multiple sclerosis. European Journal of Immunology 34:18391848. doi: https://doi.org/10.1002/eji.200324044

8. Felts PA, Woolston A-M, Fernando HB, Asquith S, Gregson NA, Mizzi OJ, Smith KJ (2005) Inflammation and primary demyelination induced by the intraspinal injection of lipopolysaccharide. Brain 128:1649-1666. doi: 10.1093/brain/awh516

9. Ferguson B, Matyszak MK, Esiri MM, Perry VH (1997) Axonal damage in acute multiple sclerosis lesions. Brain 120 ( Pt 3):393-399. doi: 10.1093/brain/120.3.393

10. Goldberg J, Clarner T, Beyer C, Kipp M (2015) Anatomical Distribution of Cuprizone-Induced Lesions in C57BL6 Mice. J Mol Neurosci 57:166-175. doi: 10.1007/s12031-015-0595-5

11. Iwahashi T, Inoue A, Koh CS, Shin TK, Kim BS (1999) Expression and potential role of inducible nitric oxide synthase in the central nervous system of Theiler's murine encephalomyelitis virusinduced demyelinating disease. Cell Immunol 194:186-193. doi: 10.1006/cimm.1999.1482

12. Jagessar SA, Kap YS, Heijmans N, van Driel N, van Straalen L, Bajramovic JJ, Brok HPM, Blezer ELA, Bauer J, Laman JD, 't Hart BA (2010) Induction of progressive demyelinating autoimmune encephalomyelitis in common marmoset monkeys using MOG34-56 peptide in incomplete freund adjuvant. J Neuropathol Exp Neurol 69:372-385. doi: 10.1097/NEN.0b013e3181d5d053

13. Licht-Mayer S, Campbell GR, Canizares M, Mehta AR, Gane AB, McGill K, Ghosh A, Fullerton A, Menezes N, Dean J, Dunham J, Al-Azki S, Pryce G, Zandee S, Zhao C, Kipp M, Smith KJ, Baker D, Altmann D, Anderton SM, Kap YS, Laman JD, Hart BA 't, Rodriguez M, Watzlawick R, Schwab JM, Carter R, Morton N, Zagnoni M, Franklin RJM, Mitchell R, Fleetwood-Walker S, Lyons DA, 
Chandran S, Lassmann H, Trapp BD, Mahad DJ (2020) Enhanced axonal response of mitochondria to demyelination offers neuroprotection: implications for multiple sclerosis. Acta Neuropathol 140:143-167. doi: 10.1007/s00401-020-02179-x

14. Mahad DH, Trapp BD, Lassmann H (2015) Pathological mechanisms in progressive multiple sclerosis. Lancet Neurol 14:183-193. doi: 10.1016/S1474-4422(14)70256-X

15. Mahad DJ, Ziabreva I, Campbell G, Lax N, White K, Hanson PS, Lassmann H, Turnbull DM (2009) Mitochondrial changes within axons in multiple sclerosis. Brain 132:1161-1174. doi: 10.1093/brain/awp046

16. McGeachy MJ, Stephens LA, Anderton SM (2005) Natural recovery and protection from autoimmune encephalomyelitis: contribution of CD4+CD25+ regulatory cells within the central nervous system. J Immunol 175:3025-3032. doi: 10.4049/jimmunol.175.5.3025

17. Mutsaers SE, Carroll WM (1998) Focal accumulation of intra-axonal mitochondria in demyelination of the cat optic nerve. Acta Neuropathol 96:139-143. doi: $10.1007 / \mathrm{s} 004010050873$

18. Nikić I, Merkler D, Sorbara C, Brinkoetter M, Kreutzfeldt M, Bareyre FM, Brück W, Bishop D, Misgeld T, Kerschensteiner M (2011) A reversible form of axon damage in experimental autoimmune encephalomyelitis and multiple sclerosis. Nat Med 17:495-499. doi: $10.1038 / \mathrm{nm} .2324$

19. Ohno N, Chiang H, Mahad DJ, Kidd GJ, Liu L, Ransohoff RM, Sheng Z-H, Komuro H, Trapp BD (2014) Mitochondrial immobilization mediated by syntaphilin facilitates survival of demyelinated axons. Proc Natl Acad Sci USA 111:9953-9958. doi: 10.1073/pnas.1401155111

20. Oleszak EL, Katsetos CD, Kuzmak J, Varadhachary A (1997) Inducible nitric oxide synthase in Theiler's murine encephalomyelitis virus infection. J Virol 71:3228-3235

21. Qi X, Lewin AS, Sun L, Hauswirth WW, Guy J (2006) Mitochondrial Protein Nitration Primes Neurodegeneration in Experimental Autoimmune Encephalomyelitis *. Journal of Biological Chemistry 281:31950-31962. doi: 10.1016/S0021-9258(19)84109-1

22. Rosenkranz SC, Shaposhnykov AA, Träger S, Engler JB, Witte ME, Roth V, Vieira V, Paauw N, Bauer S, Schwencke-Westphal C, Schubert C, Bal LC, Schattling B, Pless O, van Horssen J, Freichel M, Friese MA (2021) Enhancing mitochondrial activity in neurons protects against neurodegeneration in a mouse model of multiple sclerosis. elife 10:e61798. doi: 10.7554/eLife.61798

23. Sadeghian M, Mastrolia V, Rezaei Haddad A, Mosley A, Mullali G, Schiza D, Sajic M, Hargreaves I, Heales S, Duchen MR, Smith KJ (2016) Mitochondrial dysfunction is an important cause of neurological deficits in an inflammatory model of multiple sclerosis. Sci Rep 6:33249. doi: 10.1038/srep33249

24. Sathornsumetee S, McGavern DB, Ure DR, Rodriguez M (2000) Quantitative ultrastructural analysis of a single spinal cord demyelinated lesion predicts total lesion load, axonal loss, and neurological dysfunction in a murine model of multiple sclerosis. Am J Pathol 157:1365-1376. doi: 10.1016/S0002-9440(10)64650-0

25. Smith KJ, Lassmann $\mathrm{H}$ (2002) The role of nitric oxide in multiple sclerosis. Lancet Neurol 1:232241. doi: 10.1016/s1474-4422(02)00102-3 
26. Sorbara CD, Wagner NE, Ladwig A, Nikić I, Merkler D, Kleele T, Marinković P, Naumann R, Godinho L, Bareyre FM, Bishop D, Misgeld T, Kerschensteiner M (2014) Pervasive Axonal Transport Deficits in Multiple Sclerosis Models. Neuron 84:1183-1190. doi: 10.1016/j.neuron.2014.11.006

27. Trapp BD, Peterson J, Ransohoff RM, Rudick R, Mörk S, Bö L (1998) Axonal Transection in the Lesions of Multiple Sclerosis. New England Journal of Medicine 338:278-285. doi: 10.1056/NEJM199801293380502

28. Waxman SG (2006) Axonal conduction and injury in multiple sclerosis: the role of sodium channels. Nat Rev Neurosci 7:932-941. doi: 10.1038/nrn2023

29. Witte ME, Mahad DJ, Lassmann H, van Horssen J (2014) Mitochondrial dysfunction contributes to neurodegeneration in multiple sclerosis. Trends Mol Med 20:179-187. doi: 10.1016/j.molmed.2013.11.007

30. Woodruff RH, Franklin RJM (1999) Demyelination and remyelination of the caudal cerebellar peduncle of adult rats following stereotaxic injections of lysolecithin, ethidium bromide, and complement/anti-galactocerebroside: a comparative study. Glia 25:216-228. doi: 10.1002/(sici)1098-1136(19990201)25:3<216::aid-glia2>3.0.co;2-I

31. Zambonin J, Engeham S, Campbell GR, Ziabreva I, Beadle NE, Taylor RW, Mahad DJ (2010) Identification and investigation of mitochondria lacking cytochrome c oxidase activity in axons. Journal of Neuroscience Methods 192:115-120. doi: 10.1016/j.jneumeth.2010.07.022

32. Zambonin JL, Zhao C, Ohno N, Campbell GR, Engeham S, Ziabreva I, Schwarz N, Lee SE, Frischer JM, Turnbull DM, Trapp BD, Lassmann H, Franklin RJM, Mahad DJ (2011) Increased mitochondrial content in remyelinated axons: implications for multiple sclerosis. Brain 134:1901-1913. doi: 10.1093/brain/awr110 